\title{
A Multistrategy Approach to the Classification of Phases in Business Cycles
}

\author{
Katharina Morik and Stefan Rüping \\ Univ. Dortmund, Computer Science Department, LS VIII \\ \{morik, rueping\}@ls8.informatik.uni-dortmund.de \\ http://www-ai.cs.uni-dortmund.de
}

\begin{abstract}
The classification of business cycles is a hard and important problem. Government as well as business decisions rely on the assessment of the current business cycle. In this paper, we investigate how economists can be better supported by a combination of machine learning techniques. We have successfully applied Inductive Logic Programming (ILP). For establishing time and value intervals different discretization procedures are discussed. The rule sets learned from different experiments were analyzed with respect to correlations in order to find a concept drift or shift.
\end{abstract}

\section{Introduction}

The ups and downs of business activities have been observed for a long time It is, however, hard to capture the phenomenon by a clear definition. The National Bureau of Economic Research (NBER) defines business cycles as "recurrent sequences of altering phases of expansion and contraction in the levels of a large number of economic and financial time series." This definition points at the multi-variate nature of business cycles. It does not specify many of the modeling decisions to be made. There is still room for a variety of concepts.

- What are the indices that form a phase of the cycle? Production, employment, sales, personal income, and transfer payments are valuable indicators for cyclic economic behavior. Are there others that should be included?

- What is the appropriate number of phases in a cycle? The number of phases in a cycle varies in the various economic models from two to nine. The NBER model indicates two alternating phases. The transition from one phase to the next is given by the turning points trough and peak. In the model of the Rheinisch-Westfälisches Institut für Wirtschaftsforschung (RWI), a cycle consists of a lower turning point, an upswing, an upper turning point, and a downswing. Here, the turning points are phases that cover several months.

- Do all cycles follow the same underlying rules or has there been a drift of the rules?

There are two tasks investigated by economic theory, the prediction and the dating problem. Where the prediction of values of economic indicators is quite successful handled by macro-economic equations [6], the dating problem remains a challenge. In this paper, we tackle the dating problem: 
Dating: Given current (and past) business measurements, in which phase is the economy currently? In other words, the current measurements are to be classified as phases of a business cycle.

The dating problem is solved in the United States of America by a board of experts, the NBER. The data on German business cycles are classified by experts, as well. The aim is now to learn from these classified business data rules that state in which phase of the cycle a country is in. This task is less clearly defined than the task of predicting business activities, because business cycles themselves are basically a theoretical model to explain the variation in business data.

Linear discriminant analysis has been proposed as the baseline of empirical models ${ }^{1}$. Univariate rules were learned that used threshold values for separating phases. The accuracy of the 18 learned rules was $54 \%$ in cross validation. It has been investigated how the classification can be enhanced by the use of monthly data [8]. More sophisticated statistical models have been developed and achieved $63 \%$ accuracy [15]. The use of Hidden Markov Models led to developing two signals for an increase in the probability of a turning point [3]. The results cannot be tranformed into classification accuracy ${ }^{2}$. Also extensive experiments with other learning techniques (linear and quadratic discriminant analysis, neural networks, support vector machines) in [13] did not deliver a better accuracy. In summary, the results of statistical economy show how hard it is to classify business phases correctly.

In this paper, we investigate the applicability of inductive logic programming to the problem of dating phases of a business cycle. ILP was chosen because the results can easily be interpreted by the experts, experts are allowed to enter additional economic knowledge into the rule set, and ILP automatically selects the relevant features.

We were given quarterly data for 13 indicators concerning the German business cycle from 1955 to 1994 (see Figure 1), where each quarter had been classified as being a member of one of four phases [7]. The indicators are:

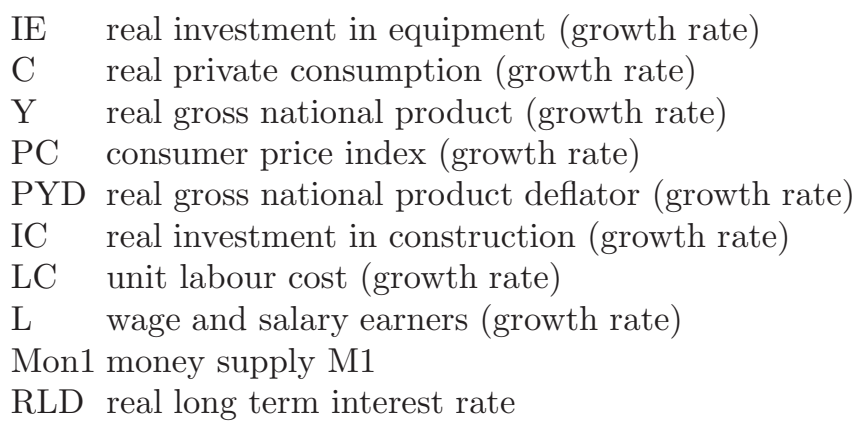

\footnotetext{
${ }^{1}$ Claus Weihs at a workshop on business cycles at the "Rheinisch-Westfälisches Institut für Wirtschaftsforschung" in January 2002

${ }^{2}$ The signals precede or follow a turning point by 5 to 7 quarters of a year [3].
} 


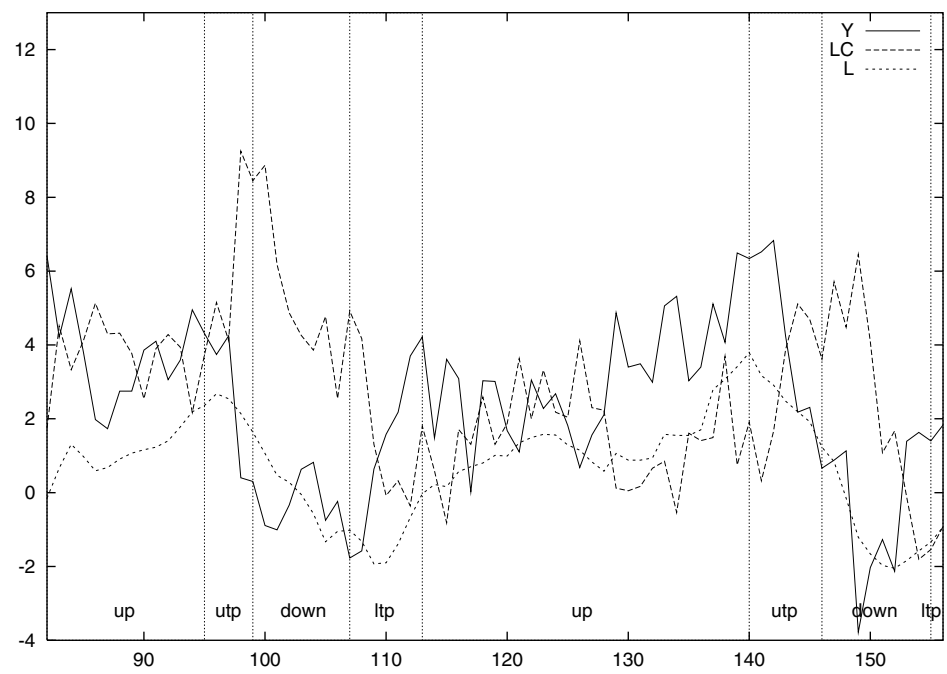

Fig. 1. Plot of the indicators $\mathrm{Y}, \mathrm{LC}$ and $\mathrm{L}$ in two successive business cycles, starting with upswing in the quarter $82(1976 / 1)$ and ending with lower turning point in the quarter $156(1994 / 3)$

RS nominal short term interest rate

GD government deficit

$\mathrm{X}$ net exports

We experimented with different discretizations of the indicator values (see Section 2.1). The discretization into ranges (levels) of values was also used in order to form time intervals. A sequence of measurements within the same range is summarized into a time interval. Relations between the different time intervals express precedence or domination of one indicator's level to another ones level. We also compared the two phase with the four phase business cycle. In summary, the following three models were inspected:

- business cycle with four phases, without time intervals, (Section 2.2)

- business cycle with four phases, with time intervals, (Section 2.3).

- business cycle with two phases, without time intervals (Section 2.4).

Particular attention was directed towards the appropriate sample size for the dating problem. The homogeneity of the data set of business cycles with two phases was investigated (Section 2.5).

\section{Experiments on German Business Cycle Data}

Our leading question was whether ILP can support economists in developing models for dating phases of the business cycle. Given the quarterly data for 
13 indicators concerning the German business cycles from 1955 to 1994 where each quarter is classified as member of one of four phases, we used all but one cycle for learning rules and tested the rules on the left-out cycle. The leave-onecycle-out test assesses the accuracy (how many of the predicted classifications of quarters corresponded to the given classification) and the coverage (how many of the quarters received a classification by the learned rules).

For ILP learning, we applied RDT [11] with the following rule schemata:

m1 (Index1, Value, Phase): $\operatorname{Index} 1(T, V), \operatorname{Value}(V) \rightarrow \operatorname{Phase}(T)$

m2 (Index1, Value,Index2, Phase):

Index $1(T, V), \operatorname{Value}(V)$, Index $2(T, V) \rightarrow \operatorname{Phase}(T)$

m3 (Index1, Value1,Index2, Value2,Phase):

Index $1(T, V 1)$, Value1(V1), Index $2(T, V 2)$, Value $2(V 2)$,

opposite $(V 1, V 2) \rightarrow \operatorname{Phase}(T)$

The predicates that can instantiate the predicate variable Index are the 13 indicators of the economy (see above). The predicates that can instantiate the predicate variable Value express the discretization of the real values of the indicators. The phase variable can be instantiated by down, ltp, up, utp for the phases of downswing, lower turning point, upswing and upper turning point of a four-phase business cycle model or by down, up for a business cycle model with two phases.

\subsection{Discretization}

The goal of discretization is to provide the learning algorithm with data in a representation from which it can generalize maximally. Actually, two discretization tasks have to be solved:

Discretization of Values: split the continuous range of possible values into finitely many discrete For example, a gross national product of 9.21 in the third quarter could be expressed as the fact $y(3, h i g h)^{3}$.

Interval Segmentation: for a given time series, find a segmentation of the time points into maximal sub-intervals, such that the values of the series in this interval share a common pattern. For example, the time series of gross national products $Y=(10.53,10.10,9.21,5.17,4.93)$ could be described as the temporal facts $y(1,3, h i g h), y(4,5$, medium $)$, but can also be described as $y(1,5$, decreasing $)$.

Interval segmentation can be viewed as discretization of the temporal values, therefore in this section we will use the name discretization as a generic term for both discretization of values and interval segmentation.

\footnotetext{
${ }^{3}$ Note, that the economic indicator $Y$ is expressed as a predicate $y$ and not as a variable in ILP.
} 
The key in interval segmentation is to find a representation, that is adequate for the learner. There are many representations for time series [12], e.g. as piecewise constant or piecewise linear functions [10], using template patterns [4] or as temporal rules [5, 9].

In our case, the data is already extensively pre-processed using economical knowledge (e.g. the gross national product was originally developed as a single indicator for the state of national economy). Also, the data is given free of trends (as growth rates). It can be assumed that the relevant information lies in the value of the indicator alone. Hence, a representation of the given time series as piecewise constant functions seems to be appropriate. This has the additional advantage, that the interval segmentation can easily be found by discretizing the attribute values and joining consecutive time points with identical discretization.

To find a high-quality value discretization, we can use the information that is given by the class of the examples in addition to the distribution of the numerical values [17]. Our goal is to find a discretization of the indicators, that already contains as much information about the cycle phase as possible. This directly leads to the use of information gain as the discretization criterion. In contrast to the usual approaches, we did not use an artificial criterion to determine the optimal number of discrete values, but used the number of interval segments that were induced by the discretization as our quality criterion. Using four discrete values usually led to a representation with a suitable number of facts. Note that this also deals with the information gains tendency to over-discretize the data, that was reported in [17].

A closer look at the resulting discretization showed that in certain cases, the indicators had a very high variation, which leads to many intervals that contained only one time point. In this case, the relevant observation may not be the value of the indicator, but the fact that this indicator was highly varying, i.e. that no definite value can be assigned to it. This can be expressed by a new fact indicator $(T 1, T 2$, unsteady), which replaces the facts indicator $(T 1, T 1+$ 1 , value $\left._{1}\right)$, indicator $\left(T 1+1, T 1+2\right.$, value $\left._{2}\right), \ldots$, indicator $\left(T 2-1, T 2\right.$, value $\left._{n}\right)$.

\subsection{Modeling Four Phases without Time Intervals}

The data correspond to six complete business cycles, each with four phases. We tested our model by a kind of leave-one-out test, where in each turn a full cycle was left out (LOO1 to LOO6). For the upper and lower turning point phases, no rule could be learned. Only for the upswing, each learning run delivered rules. For the downswing, only two learning runs, namely leaving out cycle 3 and leaving out cycle 5 , delivered rules. Misclassifications at the turning points are strikingly more frequent than in other phases. Figure 2 shows the results.

The results miss even the baseline of $54 \%$ in the average. Leaving out the fifth cycle (from 1974 until 1982) delivers the best result where both, accuracy and coverage, approach $70 \%$. This might be due to its length (32 quarters), since also in the other experiment dealing with four phases the prediction of upper turning point and upswing is best, when leaving out the fifth cycle. Since the sixth cycle is even longer (45 quarters), we would expect best results in LOO6 


\begin{tabular}{|l|l|l|l|}
\hline Cycle & Accuracy & Coverage & No.of learned rules \\
\hline LOO1 & 0.125 & 0.25 & 13 upswing \\
LOO2 & 0.5 & 1.0 & 12 upswing \\
LOO3 & 0.462 & 0.462 & 10 upswing, 2 downswing \\
LOO4 & 0.375 & 1.0 & 11 upswing \\
LOO5 & 0.696 & 0.696 & 10 uspwsing, 1 downswing \\
LOO6 & 1.0 & 0.36 & 1 upswing \\
Average & 0.526 & 0.628 & total: 60 \\
\hline
\end{tabular}

Fig. 2. Results in the four phase model using time points

I1

I2

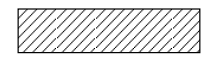

contains(I1,I2)

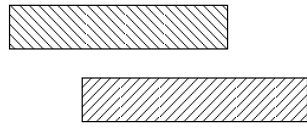

overlaps(I1,I2)

Fig. 3. The temporal relations contains and overlaps

which is true for the accuracy in this experiment. In the other experiment with four phases, the accuracy is best for upswing in LOO6 and second best for it in LOO5.

\subsection{Modeling Four Phases with Time Intervals}

Let us now see, whether time intervals can improve the results. We have used the discretization of the indicator values for the construction of time intervals (see Section 2.1). We end up with facts of the form Index (I, Range), and for each time point within the time interval $I$ a fact stating that this time point $T$ (i.e. quarter) lies in the time interval $I$ : $\operatorname{covers}(\mathrm{I}, \mathrm{T})$.

We then described the relations between different time intervals by means of Allen's temporal logic [2]. From the 13 possible relationships between time intervals, we chose contains and overlaps. The relation $\operatorname{contains}(I 1, I 2)$ denotes a larger interval $I 1$ in which somewhere the interval $I 2$ starts and ends. contains(I1, I2) is true for each time point within the larger interval I1. overlaps(I1, I2) is true for each time point of the interval $I 1$ which starts before $I 2$ is starting (see Figure 3). We left out the other possible relations, because they were either too general or too specific to be used in a classification rule or would violate the constraint, that only information about past events can be used in the classification ${ }^{4}$. The time intervals were calculated before the

\footnotetext{
${ }^{4}$ A relation that would require that the end point of one interval was identical to the starting point of another interval would be too specific. A relation that would only require that an interval would happen before another interval, regardless of the amount of time in between, would be too general.
} 
training started. The rule schemata were defined such that they link two indicators with their corresponding time intervals. One rule schema is more specialised in that it requires the time intervals of the two indicators to either overlap or include each other. This more specific rule schema was intended to find rules for the turning phases, where no rules were learned in the previous experiment. In fact, rules for the upper turning point, upswing, and downswing were learned, but no rules could be learned for the upper turning point.

Another intention behind the time interval modeling was to increase the accuracy of the learned rules. Indeed, rules for the upper turning point could be learned with the average accuracy of $75 \%$ in the leave-one-cycle-out runs. However, the accuracy for upswing decreased to $34 \%$ in the average. Hence, overall the time interval model did not enhance the results of the time point model in as much as we expected (see Table 4 ).

\begin{tabular}{|l|l|l|l|l|}
\hline Cycle & Phase & Accuracy & Coverage & No. learned rules \\
\hline LOO1 & upswing & 0.167 & 1 & 73 \\
& downswing & - & 0 & 1 \\
& utp & - & 0 & 0 \\
& ltp & - & 0 & 2 \\
\hline LOO2 & upswing & - & 0 & 103 \\
& downswing & - & 0 & 3 \\
& utp & - & 0 & 2 \\
& ltp & - & 0 & 0 \\
\hline LOO3 & upswing & 0.461 & 1 & 87 \\
& downswing & 1 & 0.200 & 2 \\
& utp & 0 & 0 & 2 \\
& ltp & - & 0 & 2 \\
\hline LOO4 & upswing & 0.167 & 1 & 59 \\
& downswing & 0.333 & 1 & 7 \\
& utp & - & 0 & 0 \\
& ltp & - & 0 & 4 \\
\hline LOO5 & upswing & 0.481 & 1 & 88 \\
& downswing & 0 & 0 & 3 \\
& utp & - & 0 & 0 \\
& ltp & 0.75 & 0.857 & 4 \\
\hline LOO6 & upswing & 0.667 & 0.296 & 6 \\
& downswing & 0.243 & 1 & 2 \\
& utp & - & 0 & 0 \\
& ltp & - & 0 & 0 \\
\hline Average & upswing & 0.388 & 0.716 & 69.3 \\
& downswing & 0.104 & 0.500 & 3 \\
& utp & 0 & 0 & 0.667 \\
& ltp & 0.75 & 0.143 & 2 \\
\hline
\end{tabular}

Fig. 4. Results in the four phase model using time intervals 


\subsection{Modeling Two Phases}

Theis and Weihs [14] have shown, that in clustering analyses of German macroeconomic data at most three clusters can be identified. The first two clusters correspond to the cycle phases of upswing and downswing and the eventual third cluster corresponds to a time period around 1971. This suggests, that two phases instead of four may be more suited for the description of business data. It also points at a concept drift (see Section 2.5). In our third experiment we mapped all time points classified as upper turning point to upswing and all quarters of a year classified as lower turning point to downswing. We then applied the rule schemata of the first experiment. An example of the learned rules is:

$i e(T, V 1), \operatorname{low}(V 1), c(T, V 2), \operatorname{high}(V 2) \rightarrow \operatorname{down}(T)$

stating that a low investment into equipment together with high private consumption indicates a downswing.

Again, leaving out the fifth or the sixth cycle gives the best results in the leave-one-cycle-out test. Accuracy and coverage are quite well balanced (see Figure 5).

These learning results are promising. They support the hypothesis that a two phase model is more appropriate for the dating task. Concerning the selection of indicators, the learning results show that all indicators contribute to the dating of the phase. However, the short term interest rate does not occur in three of the rule sets. Consumption (both the real value and the index), net exports, money supply, government deficit, and long term interest rate are missing in at least one of the learned rule sets. For the last four cycles, i.e. leaving out cycle 1 or cycle 2, some indicators predict the upswing without further conditions: high or medium number of salary earners $(l)$, high or medium investment in equipment (ie), high or medium investment in construction $(i c)$, medium consumption $(c)$, and the real gross national product $(y)$. It is interesting to note, that a medium or high real gross national product alone classifies data into the upswing phase only when leaving out cycle 1,2, or 4 . Since RDT performs a complete search, we can conclude, that in the data of cycle 1 to cycle 4 , the gross national product alone does not determine the upswing phase. Further indicators are necessary there, for instance money supply (mon 1 ) or consumer price index $(p c)$.

\begin{tabular}{|l|l|l|l|}
\hline Cycle & Accuracy & Coverage & No. learned rules \\
\hline LOO1 & 0,8125 & 0,795 & 9 upswing, 69 downswing \\
LOO2 & 0,588 & 1,0 & 17 upswing, 35 downswing \\
LOO3 & 0,823 & 0,571 & 2 upswing, 15 downswing \\
LOO4 & 0,8 & 0,35 & 6 upswing, 8 downswing \\
LOO5 & 0,869 & 0,8 & 10 upswing, 39 downswing \\
LOO6 & 1,0 & 0,701 & 6 upswing, 41 downswing \\
Average & 0,815 & 0,703 & total 50 up, 207 down \\
\hline
\end{tabular}

Fig. 5. Results in the two phase model using time points 


\subsection{Concept Shift}

Starting from the two-phase model, we analyzed the homogeneity of the business cycle data. The learning results from different leave-one-cycle-out experiments were inspected with respect to their correlation. If the same rule is learned in all experiments, this means that the underlying principle did not change over time. If, however, rules co-occur only in the first cycles or in the last cycle, we hypothesize a concept drift in business cycles. We used the correlation analysis of the APRIORI algorithm $[1,16]$.

We want to know whether some rules are learned in all training sets, or, at least, whether there are rules that are more frequently learned than others. Enumerating all learned rules we get a vector for each training set (corresponding to a transaction in APRIORI) where the learned rule is marked by 1 and the others are set to 0 . The frequency of learned rules and their co-occurrence is identified. There is no rule which was learned in all training sets. Eight rules were learned from three training sets. No co-occurrence of learned rules could be found. There is one rule, which was learned in four training sets, namely leaving out cycle 1 , cycle 4 , cycle 5 , or cycle 6 :

$\operatorname{rld}(T, V), l(T, V), \operatorname{low}(V) \rightarrow \operatorname{down}(T)$

stating that the real long term interest rate and the number of wage and salary earners being low indicates a downswing.

We now turn around the question and ask: which training sets share rules? For answering this question, a vector for each learned rule is formed where those training sets are marked by 1 which delivered the rule.

- Eighteen rules were shared in the training sets leaving out cycle 5 and leaving out cycle 6 . Four of the rules predict an upswing, fourteen rules predict a downswing. This means, that cycles 1 to 4 have the most rules in common. The data from the last quarter of 1958 until the third quarter of 1974 are more homogeneous than all the data from 1958 until 1994.

- When leaving out cycle 1 or cycle 2, eleven rules occur in both learning results. This means, that cycles 3 to 6 have second most rules in common. The data from the second quarter of 1967 until the end of 1994 are more homogeneous than all data together.

The rule set analysis shows that cycles 1 to $4(1958-1974)$ and cycles 3 to 6 (1967 - 1994) are more homogeneous than the overall data set. We wonder what happened in cycles 3 and 4 . The first oil crisis happened at the end of cycle 4 (November 1973 - March 1974). This explains the first finding well. It shows that our rule set analysis can indeed detect concept drift, where we know that a drift occured. However, the oil crisis cannot explain why cycles 3 to 6 share so many rules. The second oil crises occured within cycle $5(1979-1980)$. We assume that the actual underlying rules of business cycles may have changed over time. The concept drift seems to start in cycle 3 . The periods of cycles 1 and $2(1958-1967)$ are characterized by the reconstrucion after the world war. Investment in construction (ic) and in equipment (ie) is not indicative in this period, since it is rather high, anyway. A low number of earners $(l)$ together with 
a medium range of the gross national product deflator ( $p y d)$ best characterizes the downswing in cycles 1 to 3 - this rule has been found when leaving out cycles 4 or 5 or 6 . Since the unemployment rate was low after the war, it is particularly expressive for dating a phase in that period. This explains the second finding of our rule set analysis.

\section{Conclusion and Further Work}

ILP can be applied to the problem of classifying the phases of a business cycle with a performance that is comparable to state-of-the-art statistical methodes like linear discriminant analysis, quadratic discriminant analysis, support vector machines or neural nets [13]. There is evidence, that the high error rate, compared to other classification problems, is a result of the four phase model of business cycles. The two phase model seems to be fitting the data much better.

Machine learning techniques in concert have answered the questions that have been our starting point (see Section 1).

- ILP offers opportunities for the analysis of business cycle data. It is easy to interpret the results so that the learned rules can be easily inspected by economists. The multi-variate nature of ILP and the automatic selection of most relevant indicators fits the needs of dating problem.

- The two-phase model of the business cycle clearly outperformed the fourphase model. Where the best average accuracy in the four-phase model was $53 \%$, the average accuracy of the two-phase model was $82 \%$.

- Rule set analysis in terms of correlations between training set results shows that cycles 1-4 (1958 - 1974), leaving out cycle five or cycle six, had more rules in common than other cycles. The second most common rules were found when leaving out the first or the second cycle, i.e. with training on cycles 3-6 (1967 - 1994). Both findings can be explained in economical terms.

The results could well be further enhanced. We used discretization in a straightforward manner by creating the interval segmentation based on the discretization of values. This can be extended by using some of the work of [10, 4, 5, 9]. However, in many of these approaches it is unclear, how the resulting discretization can be interpreted. For our application understandability is a main goal.

The partitioning into two phases was very simple. A more sophisticated split within the upper and the lower turning phase, respectively, should lead to enhanced accuracy. Concept drift could be the reason for not reaching the level of accuracy that is often achieved in other domains. Training seperately cycles 4 to 6 and restricting the leave-one-cycle-out testing to these cycles could enhance the learning results.

Finally, ILP allows a close cooperation with economists, who can easily inspect the learned rules, inspect contradictions of the model to the data and add further background knowledge to the model. This makes ILP a very suitable tool for working on the validation / falsification of economical theories. 


\section{Acknowledgments}

This work has been partially sponsored by the Deutsche For-schungsgemeinschaft (DFG) collaborative research center 475 "Reduction of Complexity for Multivariate Data Structures". The authors thank Ullrich Heilemann, vice president of the Rheinisch-Westfälische Institut für Wirtschaftsforschung, for data of high quality and many valuable suggestions. We also thank Claus Weihs and Ursula Sondhauss for raising our interest in the task and providing insight in its statistical nature.

\section{References}

[1] R. Agrawal and R. Srikant. Fast algorithms for mining association rules in large data bases. In Proceedings of the 20th International Conference on Very Large Data Bases (VLDB '94), pages 478-499, Santiago, Chile, sep 1994. 315

[2] J. F. Allen. Towards a general theory of action and time. Artificial Intelligence, 23:123-154, 1984. 312

[3] Marlene Amstad. Konjunkturelle Wendepunkte: Datierung und Prognose. St.Gallen, 2000. 308

[4] Donald J. Berndt and James Clifford. Finding patterns in time series: A dynamic programming approach. In Usama M. Fayyad, Gregory Piatetsky-Shapiro, Padhraic Smyth, and Ramasamy Uthurusamy, editors, Advances in Knowledge Discovery and Data Mining, chapter 3, pages 229-248. AAAI Press/The MIT Press, Menlo Park, California, 1996. 311, 316

[5] Gautam Das, King-Ip Lin, Heikki Mannila, Gopal Renganathan, and Padhraic Smyth. Rule Discovery from Time Series. In Rakesh Agrawal, Paul E. Stolorz, and Gregory Piatetsky-Shapiro, editors, Proceedings of the Fourth International Conference on Knowledge Discovery and Data Mining (KDD-98), pages 16-22, Ney York City, 1998. AAAI Press. 311, 316

[6] Rheinisch-Westfälisches Institut für Wirtschaftsforschung. Arbeitsbericht 2000. Rheinisch-Westfälisches Institut für Wirtschaftsforschung, Essen, Germany, 2000. 307

[7] U. Heilemann and H. J. Münch. West German Business Cycles 1963-1994: A Multivariate Discriminant Analysis. CIRET-Conference in Singapore, CIRETStudien 50, 1996. 308

[8] U. Heilemann and H. J. Münch. Classification of German Business Cycles Using Monthly Data. SFB-475 Technical Reports 8/2001. Universitaet Dortmund, 2001. 308

[9] Frank Höppner. Learning temporal rules from state sequences. In Miroslav Kubat and Katharina Morik, editors, Workshop notes of the IJCAI-01 Workshop on Learning from Temporal and Spatial Data, pages 25-31, Menlo Park, CA, USA, 2001. IJCAI, AAAI Press. Held in conjunction with the International Joint Conference on Artificial Intelligence (IJCAI). 311, 316

[10] Eamonn Keogh, Selina Chu, David Hart, and Michael Pazzani. An online algorithm for segmenting time series. In Nick Cercone, T. Y. Lin, and Xindong Wu, editors, Proceedings of the 2001 IEEE International Conference on Data Mining, pages 289-296, San Jose, California, 2001. IEEE Computer Society. 311, 316 
[11] Jörg-Uwe Kietz and Stefan Wrobel. Controlling the complexity of learning in logic through syntactic and task-oriented models. Arbeitspapiere der GMD 503, GMD, mar 1991. 310

[12] Katharina Morik. The representation race - preprocessing for handling time phenomena. In Ramon López de Mántaras and Enric Plaza, editors, Proceedings of the European Conference on Machine Learning 2000 (ECML 2000), volume 1810 of Lecture Notes in Artificial Intelligence, Berlin, Heidelberg, New York, 2000. Springer Verlag Berlin. 311

[13] Ursula Sondhauss and Claus Weihs. Incorporating background knowledge for better prediction of cycle phases. Technical Report 24, Universität Dortmund, 2001. 308,316

[14] Winfried Theis and Claus Weihs. Clustering techniques for the detection of business cycles. SFB475 Technical Report 40, Universität Dortmund, 1999. 314

[15] Claus Weihs Ursula Sondhauß. Using labeled and unlabeled data to learn drifting concepts. In Miroslav Kubat and Katharina Morik, editors, Workshop notes of the IJCAI-01 Workshop on Learning from Temporal and Spatial Data, pages 38-44, Menlo Park, CA, USA, 2001. IJCAI, AAAI Press. Held in conjunction with the International Joint Conference on Artificial Intelligence (IJCAI). 308

[16] Ian Witten and Eibe Frank. Data Mining // Practical Machine Learning Tools and Techniques with JAVA Implementations. Morgan Kaufmann, 2000. 315

[17] D. A. Zighed, S. Rabaseda, R. Rakotomalala, and Feschet F. Discretization methods in supervised learning. In Encyclopedia of Computer Science and Technology, volume 40, pages 35-50. Marcel Dekker Inc., 1999. 311 